\title{
Photothermally enhanced drug release by $\kappa$-carrageenan hydrogels reinforced with multi-walled carbon nanotubes
}

\author{
Ana C. Estrada, Ana L. Daniel-da-Silva ${ }^{*}$ and Tito Trindade \\ Received (in XXX, XXX) Xth XXXXXXXXX 20XX, Accepted Xth XXXXXXXXX 20XX \\ ${ }_{5}$ DOI: $10.1039 / \mathbf{b 0 0 0 0 0 0 x}$
}

\begin{abstract}
Temperature and near infrared (NIR) light responsive multi-wall carbon nanotube (MWCNT)/K-carrageenan hydrogel composites have been prepared. The effects of the MWCNTs on the microstructure, strength, swelling and release properties of the resultant materials were investigated.

MWCNTs acted as reinforcing fillers and enhanced the mechanical properties of the hydrogels, the effect

10 being mostly nanotube concentration dependent. Surface functionalization of nanotubes had a major influence on the swelling of the composites. The increased release of a model drug (methylene blue) in in vitro conditions, from $\kappa$-carrageenan hydrogel composites due to NIR photothermal effect of MWCNTs was demonstrated at the physiological temperature. Thus, these composites are promising materials for the development of carriers for remotely activated drug delivery.
\end{abstract}

\section{1. Introduction}

In the last decade a growing number of hydrogel based nanostructured carriers for drug delivery have been developed, that compared to traditional molecular based systems offer sitespecific targeting and controlled drug release and thus minimize 20 the undesirable side effects of drugs [1,2]. One of the emerging strategies in drug delivery consists in the development of hydrogel carriers with remote controlled capabilities. The release of an encapsulated drug can be triggered remotely by external stimuli, providing a flexible control of the release rate and profile, 25 according to the specific needs of the patient $[3,4]$. Most studies on remotely triggered drug release have dealt with magnetically driven carriers $[5,6]$ upon the exposure to an alternate magnetic field. Near-infrared (NIR) light is harmless and deeply penetrates in the living tissues, hence being an attractive stimulus source for 30 remotely controlled bioapplications. However the number of studies reporting NIR activated platforms for drug delivery is still modest and mostly devoted to systems comprising gold nanostructures $[7,8]$.

Carbon nanotubes (CNTs) due to their remarkable electrical, 35 mechanical and thermal properties, have attracted a great number of research efforts in a number of different applications including energy storage [9,10] and optoelectronics [11]. Applications of CNTs in the field of medicine and biotechnology have emerged in the last few years [12-14]. Although there has been strong 40 debate about the impact of CNTs on health $[11,15,16]$ these materials have been investigated in the context of new nanomedicines due to their potential and unique properties. For example, CNTs absorb NIR light and convert the absorbed energy into heat very effectively [17]. The ability of the CNTs to 45 generate heat upon NIR irradiation is a powerful tool that has been investigated for applications such as cancer hyperthermia
[18-20] and remotely controlled drug delivery [21-23]. CNTs can therefore act as light activated nanoheaters to induce phase transitions of thermosensitive hydrogels, as recently reported for 50 agarose [23] and poly(N-isoprylacrylamide) based hydrogels [22-24]. This photothermal effect has been explored in light activated release of molecules entrapped within the hydrogel matrix [21-23].

Carrageenan comprises a family of linear water-soluble 55 sulfated polysaccharides extracted from red seaweeds. Due to their biocompatibility and ability to form thermoreversible hydrogels, carrageenan has been extensively used as gelling agent in food and pharmaceutical industries. $\kappa$-Carrageenan (figure 1) bears one esther sulphate group per disaccharide unit and presents

60 the best gelation properties within the carrageenan family. Hence in the last decade this biopolymer has been investigated as a carrier for controlled drug release in bone tissue engineering [25], gastrointestinal [26,27], vaginal [28] and ocular [29] targeted applications.

65

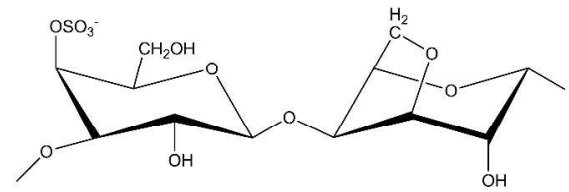

Figure 1. The structural disaccharide unit of $\kappa$-carrageenan.

$\kappa$-Carrageenan form thermoreversible gels, often stabilized by alkali-metal cations such as potassium ions. The thermo-sensitive 75 nature of $\kappa$-carrageenan hydrogels makes this biopolymer an interesting candidate for temperature activated drug delivery applications. In addition, the structure of $\kappa$-carrageenan includes a variety of chemical functional groups, providing the possibility for further derivatization and bioconjugation, as it was recently 
confirmed by the carboxymethylation of $\kappa$-carrageenan and further attachment to an antibody [30].

As reported in our recent work, the release of encapsulated molecules from $\kappa$-carrageenan hydrogels can be induced by 5 temperature due to the thermo-sensitive nature of $\kappa$-carrageenan polysaccharide [31]. Carrageenan have been proven to be effective in dispersing CNTs in water [32,33]. In principle, taken together the $\kappa$-carrageenan thermosensitive characteristics and the CNTs optical properties, the controlled release of loaded drugs in ${ }_{10} \mathrm{CNT} / \kappa$-carrageenan composites can be envisaged by irradiation with NIR light. To the best of our knowledge, the use of $\kappa-$ carrageenan for preparing NIR active carriers upon the incorporation of multi-walled carbon nanotubes (MWCNTs) is an unexplored strategy. Moreover the association of CNTs and

15 polymers of natural origin such as $\kappa$-carrageenan might be an interesting strategy to enhance the biocompatibility of CNTs.

This research demonstrates the release enhancement of a drug model (methylene blue) in in vitro conditions from $\kappa$-carrageenan hydrogels via NIR photothermal conversion of MWCNTs. $\kappa-$ 20 carrageenan nanocomposites containing pristine and functionalized MWCNTs were investigated. Additionally we have looked into the effect of the CNTs load and surface functionalization on the microstructure, strength, swelling and release properties of the hydrogel nanocomposites.

25

\section{Experimental section}

\subsection{Materials}

א-carrageenan $(300.000 \mathrm{~g} / \mathrm{mol}$ Fluka Chemie), methylene blue (C16H18CIN3S.3H2O) (Riedel-de-Haën), potassium chloride $30(\mathrm{KCl})(>99 \%$, Sigma-aldrich), phosphate buffered saline (PBS) (pH 7.4, Sigma-aldrich) and sodium azide (NaN3) (99\%, Sigmaaldrich) were used as received without any further purification. Non-functionalized MWCNTs were purchased from Nanocyl (Nanocyl-3100 series, carbon purity $>95 \%$ ).stop has been set in 35 the style to allow for easy indenting of text (although the first paragraph and paragraphs that follow headings should not be indented).

\subsection{Functionalization of MWCNTs}

40 According to the supplier, commercial MWCNTs were produced via the catalytic carbon vapor deposition (CCVD) process and have an average diameter and length of $9.5 \mathrm{~nm}$ and $1.5 \mu \mathrm{m}$ respectively. The functionalization of MWCNTs was performed following standard protocols based on the oxidation of ${ }_{45}$ commercial MWCNTs [34]. Typically, $150 \mathrm{mg}$ of MWCNTs were added to $150 \mathrm{ml}$ of $\mathrm{HNO}_{3}(65 \%$, from Fluka) and refluxed at $120{ }^{\circ} \mathrm{C}$ for $4 \mathrm{~h}$. Then mixture was cooled to room temperature and the MWCNTs were collected using filtration. The functionalized MWCNTs were then washed with distilled water 50 in order to eliminate the residual $\mathrm{HNO} 3$ and dried at $75{ }^{\circ} \mathrm{C}$ overnight. The zeta potential of the non-functionalized MWCNTs was $-6.7 \pm 0.9 \mathrm{mV}(\mathrm{pH} 4.9)$ and decreased to $-59.7 \pm 5.8 \mathrm{mV}(\mathrm{pH}$ 4.8) after functionalization. This decrease is in agreement with the introduction of ionizable acidic sites at the surface of the ${ }_{55}$ MWCNTs, such as carboxylic groups [34,35]. Further confirmation of the functionalization of MWCNTs was obtained by Fourier Transform Infrared (FTIR) spectroscopy (Figure S-1 in the Supplementary Information) and X-ray photoelectron spectroscopy (XPS) (Figures S-2, S-3 and Table S-1 in the 60 Supplementary Information).

\subsection{Preparation of MWCNT/ $/$-carrageenan nanocomposites}

The nanocomposites were prepared by blending the MWCNTs with the polymer matrix as follows. A methylene blue (MB) 0.30 $65 \mathrm{mg} / \mathrm{mL}$ aqueous solution containing the MWCNTs in the required concentration was firstly prepared. This suspension was sonicated for $1 \mathrm{~h}$ in ice bath to promote the dispersion of MWCNTs. Then $2.5 \mathrm{~mL}$ of this suspension was immediately added to $25 \mathrm{~mL}$ of a $40 \mathrm{~g} / \mathrm{L} \kappa$-carrageenan solution under magnetic stirring, at $80^{\circ} \mathrm{C}$, 70 followed by the addition of $2.5 \mathrm{~mL}$ of $\mathrm{KCl} 1 \mathrm{M}$ to promote the gelation of $\kappa$-carrageenan. Afterwards $2.5 \mathrm{~mL}$ of the composite were transferred to a cylindrical glass vial $(\varnothing 17 \mathrm{~mm})$ and allowed to cool down to room temperature to induce gelation of the composite. The gel samples were frozen at $-5^{\circ} \mathrm{C}$ over $24 \mathrm{~h}$ and 75 then lyophilized. A series of freeze dried discs of $15 \mathrm{~mm}$ diameter and $8 \mathrm{~mm}$ height were finally obtained as specimens for this research.

The synthesis conditions for the carrageenan carriers here prepared are listed in Table 1. Non-functionalized and 80 functionalized MWCNTs in variable amounts $(0.025$ and 0.25 $\mathrm{wt} \%$ based on polymer content) were used as the dispersed components in the nanocomposites. For comparison purposes, carriers containing carrageenan, potassium ions and $\mathrm{MB}$ were also prepared following the procedure described above but 85 without MWCNTs as the dispersed phase (blank hydrogel).

Table 1. Carrageenan hydrogels and corresponding synthesis conditions.

\begin{tabular}{ccc}
\hline $\begin{array}{c}\text { Sample } \\
\text { designation }\end{array}$ & Nanofiller & $\begin{array}{c}\mathbf{\%} \mathbf{w} / \mathbf{w} \\
\mathbf{m}_{\mathbf{C N T s}} / \mathbf{m}_{\text {polymer }}\end{array}$ \\
\hline blank & - & 0 \\
\hline CNT-0.025 & Non-functionalized & 0.025 \\
CNT-0.25 & MWCNT & 0.25 \\
\hline f-CNT-0.025 & Functionalized & 0.025 \\
f-CNT-0.25 & MWCNT & 0.25 \\
\hline
\end{tabular}

\section{${ }_{90}$ 2.4. Swelling studies}

The swelling measurements were carried out by immersion of lyophilized hydrogel discs in PBS $0.01 \mathrm{M} \mathrm{pH} 7.4$ at $37^{\circ} \mathrm{C}$. At the required intervals of time, the samples were removed from the solution and wiped with filter paper to remove the excess of 95 water before being weighted. The swelling ratio (Q) was calculated from equation (1):

$Q=\frac{W_{s}-W_{d}}{W_{d_{100}}}$

where $\mathrm{Wd}$ and $\mathrm{Ws}$ is the weight of the lyophilized and swollen gel, respectively. The equilibrium swelling ratio (Qequil) was determined at the point the hydrated gels achieved a constant weight value. The experiments were performed in triplicate. 


\subsection{Direct visual observation of photoinduced gel-sol transition}

Digital photographs were taken for samples of MWCNT-polymer hydrogel nanocomposites $(1 \mathrm{~mL})$ placed in vials and directly 5 irradiated with a NIR lamp (Reflector R80, 120W, $1200 \mathrm{~nm}$, Leuci) located at a distance of $8 \mathrm{~cm}$ above the samples for 3.5 min. $\kappa$-carrageenan gels without MWCNTs were also irradiated as control samples. The temperature of the samples was measured with a mercury thermometer (measurements performed with NIR 10 lamp off).

\subsection{In vitro $M B$ release studies}

Methylene blue was used as a model drug and was loaded during the stage of the preparation of the nanocomposites as described 15 above. MB has been used as a model drug namely because it is a water-soluble dye that allows an immediate visual inspection of the test. The release experiments were performed in a thermostatic orbital shaker KS 4000I Control from IKA at $37^{\circ} \mathrm{C}$ and $120 \mathrm{rpm}$, with and without NIR light irradiation. For the 20 experiments performed under NIR exposure the samples were irradiated using a NIR lamp (Reflector R80, $120 \mathrm{~W}$ and $75 \mathrm{~W}$, $1200 \mathrm{~nm}$, Leuci) located at $21 \mathrm{~cm}$ above the samples. The variation of the temperature of the release medium with light exposure time was measured during the experiment. A 25 lyophilized disc was introduced in a glass beaker containing 50 $\mathrm{mL}$ of PBS $0.01 \mathrm{M}$ at $\mathrm{pH}=7.4$ and $0.05 \%(\mathrm{w} / \mathrm{v})$ sodium azide as preservative. After predetermined intervals, $1.0 \mathrm{~mL}$ of the release medium was drawn and analyzed by UV-Vis spectroscopy $(\lambda=$ $663 \mathrm{~nm}$ ) to determine the amount of MB released at each time 30 point and replaced by $1 \mathrm{~mL}$ of fresh $\mathrm{PBS}$ to maintain the original volume. Prior to UV-Vis analysis the aliquot was diluted into $\mathrm{KCl} 1 \mathrm{M}$ (dilution ratio 1:6) to ensure that the MB released did not interact with $\kappa$-carrageenan [36]. The cumulative released fraction at time $\mathrm{t}(\mathrm{mt} / \mathrm{m} 0)$ was calculated using the equation (2):

35

$\frac{m_{t}}{m_{0}}=\frac{50 \times C_{n}+\sum_{i=0}^{n-1} C_{i}}{m_{0}}$

where $\mathrm{mt}$ is the cumulative mass of $\mathrm{MB}$ released at time $\mathrm{t}, \mathrm{m} 0$ is 40 the initial mass of loaded $\mathrm{MB}, \mathrm{Ci}$ is the mass concentration of $\mathrm{MB}$ (per $\mathrm{mL}$ ) of the aliquot, $\mathrm{Cn}$ is the mass concentration of $\mathrm{MB}$ (per $\mathrm{mL}$ ) of the aliquot at time $\mathrm{t}$ and $\mathrm{n}$ is the total number of aliquots extracted until time $t$.

\section{${ }_{45}$ 2.7. Instrumentation}

FTIR spectra of carrageenan and carrageenan nanocomposites were collected using a spectrometer Mattson 7000 (USA) coupled to a horizontal attenuated total reflectance (ATR) cell, accumulating 256 scans and using a resolution of $4 \mathrm{~cm}-1$. A ${ }_{50}$ Jasco V 560 Ultra-violet/Visible (UV/Vis) spectrophotometer (Jasco Inc., USA) was used for recording the UV/Vis absorption spectra of the aliquots. SEM analysis of blank hydrogel and carrageenan nanocomposites was performed using a scanning electron microscope Hitachi SU-70 at an accelerating voltage of

${ }_{55} 15 \mathrm{kV}$, using carbon sputtered samples. The surface charge of the non-functionalized and functionalized MWCNTs was assessed by zeta potential measurements, using a Zetasizer Nanoseries instrument from Malvern Instruments (UK). The dynamic mechanical analysis (DMA) measurements of blank hydrogel and 60 carrageenan nanocomposites were performed on a Tritec 2000 DMA dynamic mechanical analyser (Triton Technology Ltd., UK). The hydrogels were clamped between a parallel-plate compression clamp and an oscillatory deformation with amplitude of $10 \mu \mathrm{m}$ and a frequency of $1 \mathrm{~Hz}$ was applied at ${ }_{65}$ various static forces ranging from 0.1 to $0.5 \mathrm{~N}$. The elastic modulus was calculated from extrapolation of experimental data towards zero compression conditions as described elsewhere [37]. DMA measurements were performed in triplicate. The gel-sol transitions of $\kappa$-carrageenan hydrogels were determined by 70 differential scanning calorimetry (DSC) using a Shimadzu DSC50 calorimeter. $30 \mu \mathrm{L}$ aluminum pans were used with sample masses of ca. $25 \mathrm{mg}$. Hydrogel samples were heated from $25^{\circ} \mathrm{C}$ to $80{ }^{\circ} \mathrm{C}$ at $2{ }^{\circ} \mathrm{C}$ min- 1 . An empty pan was used as reference. The XPS analysis of carbon nanotubes was performed using an 75 ESCALAB 200A, VG Scientific (UK) with PISCES software for data acquisition and analysis. For analysis, an achromatic $\mathrm{Al}(\mathrm{Ka})$ X-ray source operating at $15 \mathrm{kV}(300 \mathrm{~W})$ was used, and the spectrometer, calibrated with reference to $\mathrm{Ag} 3 \mathrm{~d} 5 / 2(368.27 \mathrm{eV})$, was operated in CAE mode with $20 \mathrm{eV}$ pass energy. Data 80 acquisition was performed with a pressure lower them 1.E-6 Pa. Spectra analysis was performed using peak fitting with GaussianLorentzian peak shape and Shirley type background subtraction (or linear taking in account the data).

\section{${ }_{85}$ 3. Results and Discussion}

\subsection{Structure and mechanical properties of $\mathrm{MWCNT} / \kappa-$ carrageenan composites}

MWCNT/א-carrageenan nanocomposites were prepared by blending the components and MWCNTs were dispersed by 90 ultrasound technique. This led to $\kappa$-carrageenan suspensions of MWCNTs (both pristine and functionalized) which were macroscopically homogeneous and without visible aggregates. Figure 2 shows representative FTIR-ATR spectra of lyophilized nanocomposites containing $0.25 \mathrm{wt} \%$ of non-functionalized 95 (CTN-0.25) and functionalized (f-CNT-0.25) MWCNTs.

100

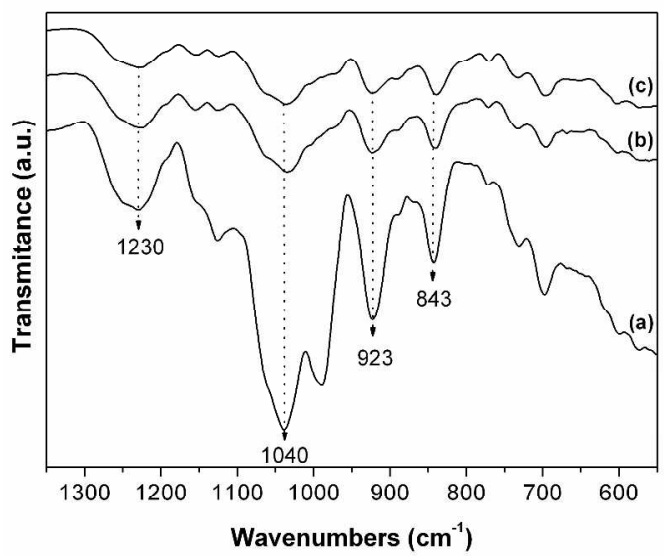

110 Figure 2. ATR-FTIR spectra of (a) $\kappa$-carrageenan and corresponding MWCNT composites: (b) CNT-0.25 and (c) f-CNT-0.25. 
All samples showed the typical absorptions bands of $\kappa$ carrageenan polysaccharide: $\approx 1225 \mathrm{~cm}^{-1}, \mathrm{~S}-\mathrm{O}$ asymmetric sulfate stretch; 1010-1070 $\mathrm{cm}^{-1}, \mathrm{C}-\mathrm{O}$ and $\mathrm{C}-\mathrm{OH}$ stretching and $843 \mathrm{~cm}^{-}$ ${ }^{1}, \alpha(1,3)$-D-galactose C-O-S stretch [38] thus indicating that the 5 biopolymer chains maintained their chemical characteristics when in contact with the MWCNTs.

In order to investigate the effect of MWCNTs in the viscoelastic properties of the hydrogel, the elastic modulus (E') of the hydrogels was assessed by DMA measurements (Table 2).

10

Table 2. The elastic modulus (E') and equilibrium swelling ratio (Qequil) of blank hydrogel and MWCNT nanocomposites.

\begin{tabular}{ccc}
\hline Hydrogel & $\mathbf{E}^{\prime} \mathbf{( k P a )}$ & $\mathbf{Q}_{\text {equil }}$ \\
\hline Blank & $147.0 \pm 11.2$ & $21.8 \pm 1.8$ \\
CNT-0.025 & $196.3 \pm 14.9$ & $16.7 \pm 1.9$ \\
CNT-0.25 & $223.2 \pm 13.8$ & $18.4 \pm 0.6$ \\
f-CNT-0.025 & $202.4 \pm 10.2$ & $29.9 \pm 2.4$ \\
f-CNT-0.25 & $229.4 \pm 1.0$ & $25.5 \pm 0.4$ \\
\hline
\end{tabular}

Overall the nanocomposites exhibit higher E' values than the 15 blank hydrogel and the E' values increase with increasing content of MWCNTs. The enhancement of the elastic moduli indicates that the presence of MWCNTs, even for the low amount present in these samples, promotes the formation of stronger $\kappa$ carrageenan hydrogels. The elastic moduli of the blank hydrogel 20 increases ca. $34 \%$ with the addition of $0.025 \mathrm{wt} \%$ of nonfunctionalized MWCNTs (CNT-0.025) and ca. 52\% at a concentration of 0.25 wt $\% \quad(\mathrm{CNT}-0.25)$. The observed enhancement of E' is slightly higher when functionalized MWCNTs were used as nanofillers; the increment of E' was ca. ${ }_{25} 38 \%$ for a concentration of funcionalized nanotubes of $0.025 \mathrm{wt} \%$ (f-CNT-0.025) and ca. 56\% for a concentration of $0.25 \mathrm{wt} \%$ (fCNT-0.25). The covalent functionalization is expected to improve the dispersion of the MWCNTs in the polymer and to favour interactions between nanotubes and polymers, thus leading 30 to a more effective load transfer from the polymer to the nanotubes and consequently enhanced mechanical properties [39].

SEM images of the lyophilized hydrogels show a continuous structure comprising polymer flakes and irregular pores with high 35 interconnectivity (Figure 3). The gelation mechanism of $\kappa-$ carrageenan involves a coil to helix conformational transition, followed by helix aggregation to form an infinite threedimensional network [40]. Thus, the flakes observed by SEM are most likely formed by aggregates of $\kappa$-carrageenan helices. After 40 incorporation of non-functionalized (Figure 3(b)) and functionalized (Figure 3(c)) MWCNTs, the carrageenan flakes appear larger, thus suggesting that the nanotubes have promoted the association of carrageenan helices in larger extension, which is in agreement with the DMA results discussed above.

${ }_{45}$ Hydrogels whose range thermo-sensitivity is slightly higher than $37{ }^{\circ} \mathrm{C}$ and up to about $44^{\circ} \mathrm{C}$ are required for their use as thermally activated drug carriers, because this is the temperature range acceptable for living cells. Therefore, the potential of the MWCNT/carrageenan composites as thermosensitive carriers was 50 first assessed by measuring the gel-sol transition by DSC. Figure 4 shows the DSC thermograms for the carrageenan gel and nanocomposites.
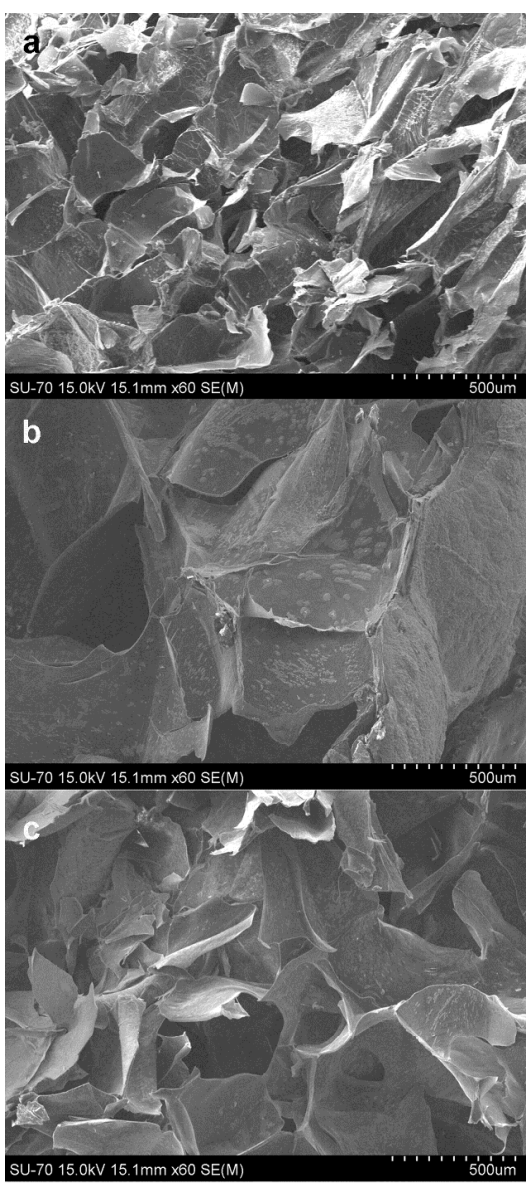

Figure 3. SEM micrographs of lyophilized (a) blank hydrogel and nanocomposites $(0.25 \mathrm{wt} \%)$ prepared with (b) non-functionalized 85 and (c) functionalized MWCNTs.

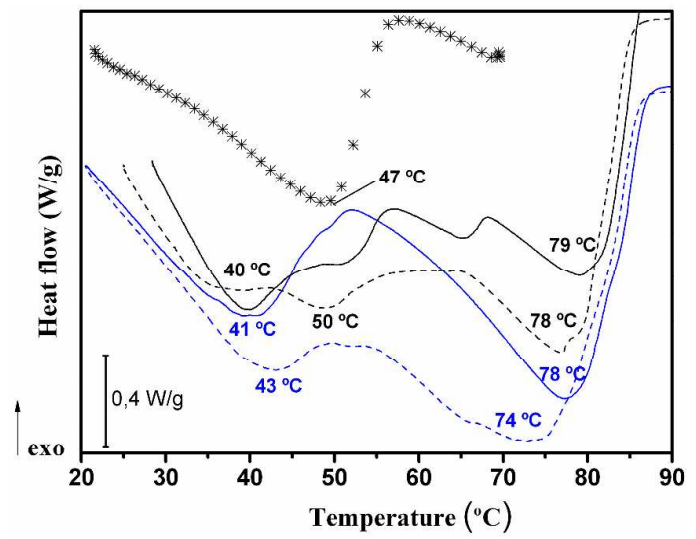

Figure 4. DSC thermograms recorded during heating of blank 90 hydrogel (stars) and composites containing non-functionalized (black lines) and functionalized (blue lines) MWCNTs at a concentration of $0.025 \mathrm{wt} \%$ (dashed lines) and $0.25 \mathrm{wt} \%$ (full lines).

95 The DSC thermogram of $\kappa$-carrageenan hydrogel (Figure 4) shows a broad endothermic peak centred at ca. $47^{\circ} \mathrm{C}$ corresponding to the melting (gel-to-sol transition) of the hydrogel [30]. The melting of $\kappa$-carrageenan hydrogels is known 
to involve the breaking up of aggregates of double helical conformations which act as physical junctions in the gel [40]. For the MWCNT composites two main endothermic peaks are observed in Figure 4. The first peak is centred at ca. $40-50^{\circ} \mathrm{C}$, 5 while the second peak is located at high temperature, in the range $75-80^{\circ} \mathrm{C}$. The occurrence of peak splitting in the DSC thermogram of the hydrogels was previously reported for $\kappa$ carrageenan gels containing $\mathrm{NaI}$ and CsI salts [41] and in $\kappa$ carrageenan hydrogel nanocomposites filled with magnetite 10 nanoparticles [30]. In these cases the gel network comprises carrageenan helices and aggregates having various sizes that undergo melting at different temperatures. Therefore, a possible explanation for our observations relies on the association of carrageenan helices around MWCNTs leading to an

15 heterogeneous gel network. This effect could be in this case even more pronounced due to the anisotropy of the fillers. In fact, an identical behavior was observed in $\kappa$-carrageenan hydrogels containing other anisotropic nanoparticles such as gold nanorods [42].

20 The above DSC results show that MWCNT/ $/$-carrageenan hydrogel nanocomposites are thermo-sensitive between $37-44^{\circ} \mathrm{C}$ and do not undergo complete transition to sol state within this temperature range. Therefore these properties offer the possibility of applications in thermally controlled delivery of loaded drugs.

25

\subsection{Swelling properties}

The equilibrium swelling ratio (Qequil) for the blank $\kappa$ carrageenan hydrogel and the MWCNT nanocomposites are listed in Table 2. For pure $\kappa$-carrageenan hydrogel a Qequil value 30 equal to $21.8 \pm 1.8$ was found. The incorporation of nonfunctionalized MWCNTs produced composites with lower Qequil values than the blank hydrogel. This is most likely due to the reinforcement of the hydrogel structure promoted by these nanotubes, as confirmed by the enhancement of the elastic moduli

35 of the composites (Table 2). The formation of a tighter gel network should oppose the swelling of the gel. Conversely, hydrogels filled with functionalized MWCNTs show higher Qequil values than the blank hydrogel despite having E' values similar to those of the composites prepared with non40 functionalized MWCNTs. This is an interesting system in which the hydrogel swelling rate seems to result from a balance between the mechanical reinforcement role of the MWCNTs fillers and surface chemistry effects. For the functionalized MWCNTs, the results are in agreement with an increase of the afflux of water

45 into the $\kappa$-carrageenan hydrogels due to the presence of polar carboxylic groups at the surface of the MWCNTs.

\subsection{Photoinduced phase transition of $\kappa$-carrageenan hydrogels}

${ }_{50}$ Samples of the hydrogels $\kappa$-carrageenan alone and composites with nanotubes were exposed to NIR light (Figure 5).

Under NIR-light irradiation, the MWCNT/א-carrageenan hydrogel composites (0.25 wt $\%$ CNTs) exhibit a gel-to-sol transition after $3.5 \mathrm{~min}$ exposure, while for the unloaded $\kappa$ 55 carrageenan (blank) and the composites containing low CNTs content $(0.025 \mathrm{wt} \%)$ did not undergo any visible phase transition. After 3.5 min light irradiation, the temperature of the composite gels $(0.025 \mathrm{wt} \%)$ have increased two degrees as compared to the

60

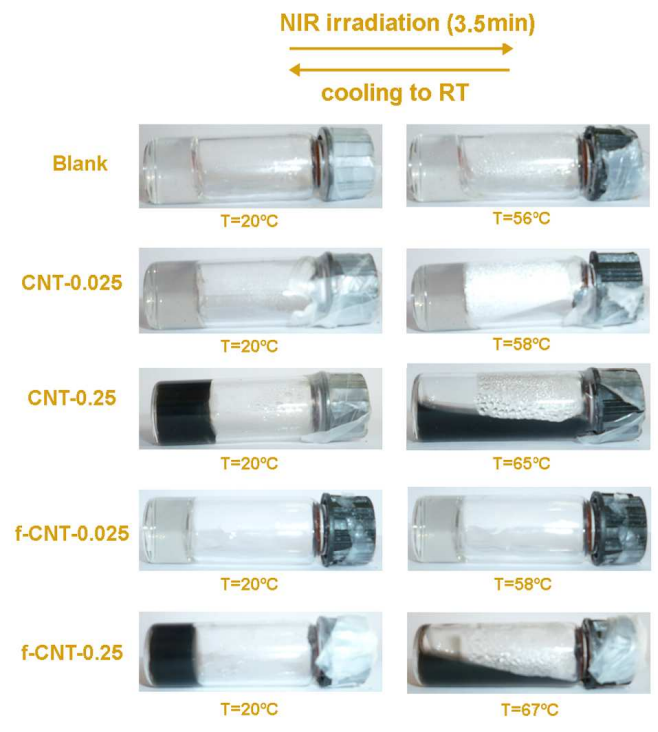

Figure 5. Visual observation of the photoinduced gel-sol ${ }_{65}$ transition in samples of $\kappa$-carrageenan-MWCNT hydrogel nanocomposites (all gels were distant $8 \mathrm{~cm}$ from the lamp).

blank hydrogel. However, figure 5 shows that this temperature increment did not cause a noticeable transition in the gel. For 70 high CNTs content $(0.25 \mathrm{wt} \%)$ the temperature increased from $20^{\circ} \mathrm{C}$ to $65^{\circ} \mathrm{C}$ and $67^{\circ} \mathrm{C}$, for gels containing pristine and functionalized MWCNTs respectively, i.e. about $10^{\circ} \mathrm{C}$ higher than the temperature increase observed in the blank hydrogel. These observations show that both pristine and functionalized ${ }_{75}$ MWCNTs act as photoinduced heaters. Noteworthy, the heat generated cause the gel-to-sol transition of the $\kappa$-carrageenan gels for MWCNTs concentrations superior to a certain threshold. Note that at this range of temperatures $\left(65-67^{\circ} \mathrm{C}\right)$ the composites were still rather viscous most probably because not all the double 80 helical aggregates of carrageenan were broken up at this temperature, which is in agreement with DSC thermograms (Figure 4). The gel-to-sol transition was reversible after incubation at room temperature. These results are in line with previous observations on the NIR light induced gel-to-sol 85 transition in SWCNT/agarose composite hydrogels [23].

\subsection{MB release profiles}

The amount of released $\mathrm{MB}$ was monitored by measuring the absorbance of the release medium at $663 \mathrm{~nm}$, i.e. at the 90 wavelength of maximum absorbance for $\mathrm{MB}$. The release experiments were performed at $37^{\circ} \mathrm{C}$ with and without exposure to NIR light. The effects of the load and functionalization of MWCNTs on the MB release profiles were then investigated.

\section{3.4.1. Temperature induced release experiments}

Figure 6 displays the release profiles of MB in PBS from $\kappa$ carrageenan blank hydrogel and nanocomposites containing non-functionalized and functionalized MWCNTs at variable loads. These release experiments were performed in isothermal 
conditions $\left(37^{\circ} \mathrm{C}\right)$ without exposure to NIR light.

All the hydrogels studied here have shown a sustainable MB release for at least 30 hours. The composites showed slower MB release than in the blank hydrogel. For $0.025 \mathrm{wt} \%$ content in ${ }_{5}$ CNTs, the MB was released at a rate slightly lower than in the blank hydrogel and no striking differences arose from the functionalization of CNTs.

10

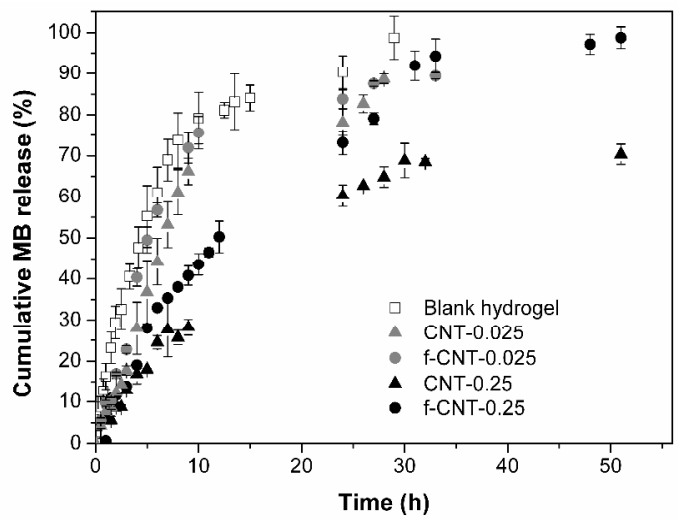

Figure 6. In vitro $\mathrm{MB}$ release profiles in $\mathrm{PBS}$ at $37^{\circ} \mathrm{C}$ from the blank hydrogel and hydrogel nanocomposites containing variable loads of non-functionalized and functionalized MWCNTs.

15 For CNT load 10 times higher $(0.25 \mathrm{wt} \%)$ the MB releases was significantly slower than in the blank hydrogel. This trend is in line with the enhancement of the elastic moduli of the hydrogels with increased content of CNTs (Table 2), stronger gels resulting in slower MB release. Previous observations reporting the release 20 kinetics in $\kappa$-carrageenan gels with variable strength but tailored by varying the counter ion concentration [43] and nature [44], also support these findings. In the first period of 5 hours the MB release profile from both non-functionalized and functionalized MWCNTs composites are identical. After this period of time the ${ }_{25} \mathrm{MB}$ release from $\mathrm{f}-\mathrm{CNT}$ composites has increased in relation to the release from the non-functionalized MWCNTs. The maximum of MB released in f-CNT composites was ca. $95 \%$ in opposition to ca. $65 \%$ in the composites with the nonfunctionalized MWCNTs. Although these composites have 30 similar E' values, thus similar MB release profiles would be expected, the differences observed account for the distinct swelling behavior. As previously discussed (Table 2), the composites containing functionalized MWCNTs achieve higher equilibrium swelling ratio than those prepared with non 35 functionalized MWCNTs. Hence the transport of the MB from the matrix to the surrounding medium is facilitated in the swollen matrix.

\subsubsection{Photoinduced release experiments}

40 Figure 7 compares the release patterns of MB in PBS from $\kappa$ carrageenan alone and from composite discs obtained with and without exposure to NIR light.

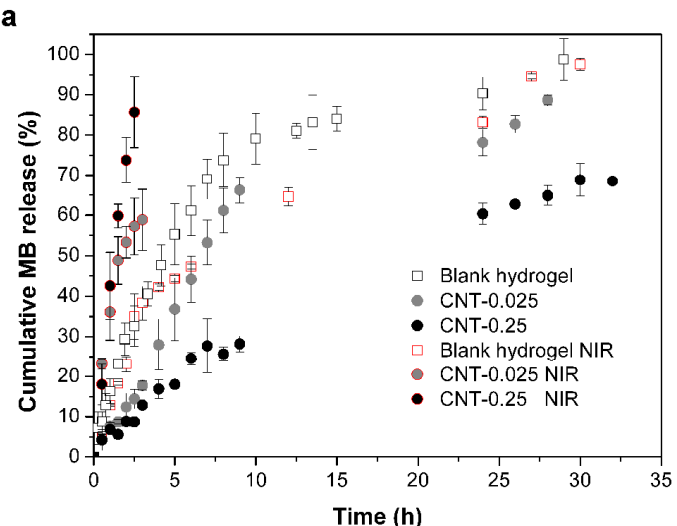

60

b

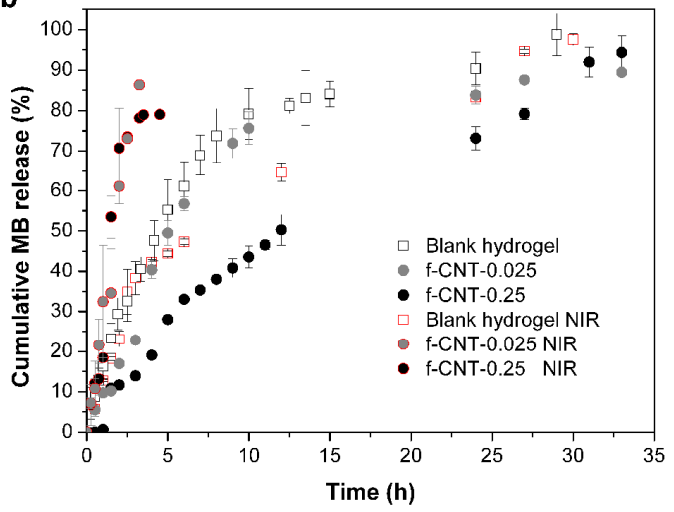
75 80 85

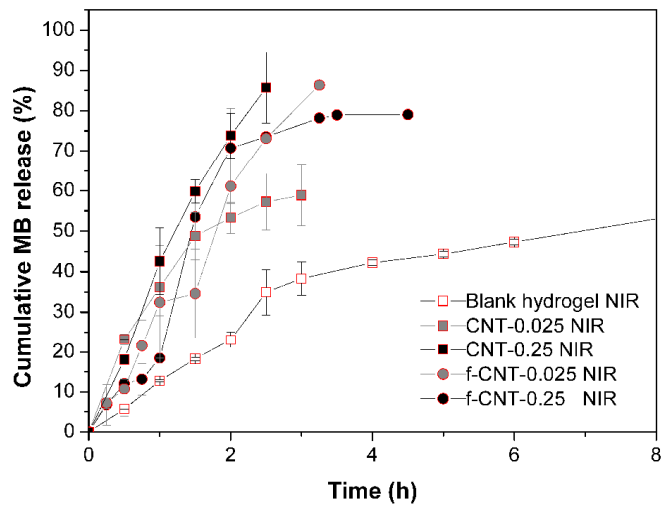

Figure 7. In vitro $\mathrm{MB}$ release profiles in $\mathrm{PBS}$ at $37^{\circ} \mathrm{C}$ from the blank hydrogel and hydrogel nanocomposites containing variable loads of (a) non-functionalized MWCNTs and (b) functionalized 90 MWCNTs exposed to NIR light $120 \mathrm{~W}$ (profiles obtained without exposure to NIR light are also displayed for comparison); (c) detail of the release profiles upon NIR light, for the first 8 hours (lines are included for eye guidance).

${ }_{95}$ Figure 7a shows that, under exposure to NIR light, the MB is released from the composites containing non-functionalized MWCNTs much faster than in the blank hydrogel, in opposition to the behavior displayed without exposure to NIR light. The irradiation of NIR light accelerates significantly the MB release 100 from these composites and this effect seems to be more marked for increasing amount of nanotubes. An identical effect was observed in the composites containing functionalized MWCNTs, although the influence of CNTs amount was less noticeable (Figure $7 \mathrm{~b}$ and $7 \mathrm{c}$ ). Conversely the effect of irradiating NIR light 
has a minor effect on the release profile of $\kappa$-carrageenan alone (blank hydrogel). Thus, these results suggest that the NIR light induced heat generation from the MWCNTs resulted in a faster release of MB from hydrogels, most likely because it promotes ${ }_{5}$ the transition from gel to sol of these temperature sensitive hydrogels.

At this point we note that, although the release experiments have been performed with a thermostatic orbital shaker set at $37^{\circ} \mathrm{C}$, NIR irradiation might cause a slight increase of the 10 temperature of the release medium due to photon absorption by water molecules at the light wavelength used in this study (1200 $\mathrm{nm})$ [45]. Hence the temperature of the release medium was monitored during the experiments and it was found that it could increase up to $42^{\circ} \mathrm{C}$, a temperature still acceptable for living cells.

${ }_{15}$ For comparison, the MB release was also investigated at these temperatures, without exposing to NIR light (Figure S-4 in the Supplementary Information). These control experiments clearly showed that such temperature increment in the release medium had a minor impact on the MB release profile and could not be 20 the main cause for the acceleration of the MB release from the composites when irradiated with NIR light.

Based solely on the differences in the temperature rise of the hydrogel composites upon NIR irradiation (Figure 5), we would expect a faster MB release by increasing the CNTs amount from ${ }_{25} 0.025 \mathrm{wt} \%$ to $0.25 \mathrm{wt} \%$. On the contrary, differences on the release rate between composites prepared with these two CNTs loads are minor when using functionalized CNTs and noticeable only after $2 \mathrm{~h}$ release when using pristine CNTs (Figure 7c). A possible explanation relies on the changes of the hydrogel ${ }_{30}$ properties arising from the incorporation of the CNTs. Indeed, as noticed in Table 2, such increase of the CNTs amount also rendered hydrogels with enhanced elastic modulus, which will have the opposite effect on the MB release than the additional heat generation from CNTs. Hence, we might conclude that the 35 rate of the NIR light induced MB release will result from the balance between the temperature rise and the mechanical properties of the hydrogels, both being affected by the concentration of the CNTs.

Aiming to unveil the effect of CNTs amount on the photo-thermal 40 induced release, additional experiments were conducted with a reduced level of NIR irradiation $(75 \mathrm{~W})$. When reducing the power of NIR lamp from $120 \mathrm{~W}$ to $75 \mathrm{~W}$, the release from the composites with functionalized CNTs decelerate, this effect being more evident in the hydrogels containing less CNTs (Figure 8). 45 Indeed, at reduced irradiation, differences in the release rate with CNTs content are clear. The results indicate that for low CNTs content $(0.025 \mathrm{wt} \%)$ and low power lamp, the hydrogel temperature increment that arises from the photo-thermal effect is little and has a small effect on the release rate, whereas for CNTs 50 amount 10 times higher $(0.25 \mathrm{wt} \%)$ the heat generated accelerates significantly the MB release when compared to the blank hydrogel.

55

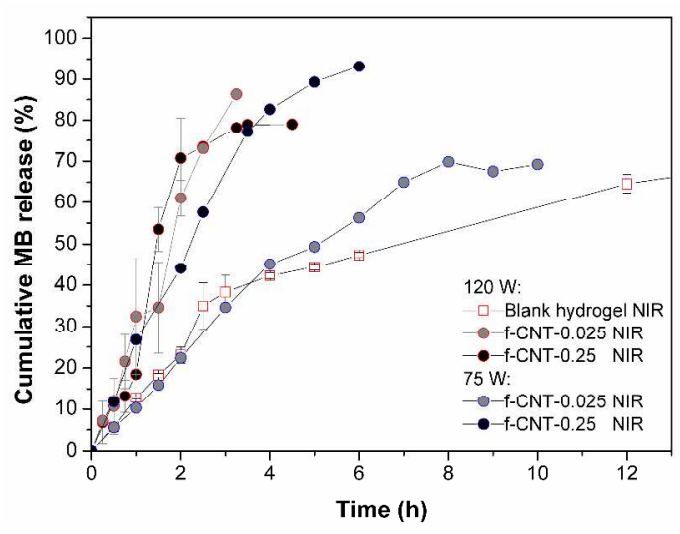

Figure 8. In vitro $\mathrm{MB}$ release profiles in $\mathrm{PBS}$ at $37^{\circ} \mathrm{C}$ from the ${ }_{60}$ hydrogel nanocomposites containing variable loads of functionalized MWCNTs exposed to NIR light from a $75 \mathrm{~W}$ lamp. Profiles obtained using a NIR $120 \mathrm{~W}$ lamp are also displayed for comparison (lines are included for eye guidance).

\section{${ }_{65}$ Conclusions}

We have succeeded in fabricating light responsive $\kappa$-carrageenan hydrogels upon the incorporation of MWCNTs. MWCNTs acted as multifunctional fillers by enhancing the mechanical properties of the hydrogels and conferring light responsive characteristics to 70 the composites. The composite hydrogels ( $0.25 \mathrm{wt} \%$ MWCNTs) showed reversible heat induced gel-to-sol phase transition, either by using temperature or NIR light irradiation as external stimuli. The release of a model molecule (methylene blue) from the hydrogels containing nanotubes could be induced by NIR 75 irradiation, in which the MWCNTs acted as a nanoheater to raise the local temperature of the gel via the photothermal conversion of the MWCNTs. In face of these promising results we believe that these hydrogel composites have potential in the development of remotely controlled light activated drug delivery systems.

80

\section{Acknowledgements}

The authors acknowledge FCT (Pest-C/CTM/LA0011/2011, SFRH/BPD/86780/2012), FSE and POPH for funding. We thank the RNME (National Electronic Microscopy Network) for SEM 85 facilities and the National Program for Scientific Equipment Renewal (REED/515/CTM/2005) for DMA measurements. The authors are very grateful to Dr. A. V. Girão, MSc. M. C. Azevedo and MSc. S. Magina for technical support.

\section{Notes and references}

90 * Department of Chemistry, CICECO, Aveiro Institute of Nanotechnology, University of Aveiro, 3810-193 Aveiro, Portugal. Fax +351 234370 084; Tel +351234370368; E-mail ana.luisa@ua.pt $\dagger$ Electronic Supplementary Information (ESI) available: [Supplementary material (FTIR and XPS spectra of functionalized MWCNTs; effect of the 95 temperature of the release medium on the MB release) is available in the online version of this article.]. See DOI: $10.1039 / \mathrm{b} 000000 \mathrm{x} /$

1 M. Hamidi, A. Azadi, P. Rafiei, Adv. Drug Delivery Rev., 2008, 60, 1638.

1002 T. R. Hoare, D. S. Kohane, Polymer, 2008, 49, 1993. 
3 N. S. Satarkar, D. Biswal, J. Z. Hilt, Soft Matter, 2010, 6, 2361.

4 B. P. Timko, T. Dvir, D. S. Kohane. Adv. Mater., 2010, 22, 4925.

5 C. S. S. R. Kumar, F. Mohammad, Adv. Drug Delivery Rev., 2011, 63, 789 .

56 C. S. Brazel, Pharm. Res., 2009, 26, 644.

7 M. P. Melancon, M. Zhou, C. Li, Accounts Chem. Res., 2011, 44, 947.

8 M. S. Yavuz, Y. Cheng, J. Chen, C. M. Cobley, Q. Zhang, M. Rycenga, J. Xie, C. Kim, K. H. Song, A. G. Schwartz, L. V. Wang, Nat. Mater., 2009, 8, 935.

9 Z. Chen, D. Zhang, X. Wang, X. Jia, F. Wei, H. Li, Y. Lu, $A d v$. Mater., 2012, 24, 2030.

10 E. Frackowiak, F. Beguin, Carbon, 2002, 40, 1775.

11 P. Avouris, M. Freitag, V. Perebeinos, Nat. Photonics, 2008, $2,341$.

1512 Z. Liu, S. Tabakman, K. Welsher, H. Dai, Nano. Res., 2009, $2,85$.

13 K. Kostarelos, A. Bianco, M. Prato, Nat. Nanotechnol., 2009, 4, 627.

14 S. K. Vashist, D. Zheng, G. Pastorin, K. Al-Rubeaan, J. H. T. Luong, F-S. Sheu, Carbon, 2011, 49, 4077.

15 C. P. Firme, P. R. Bandaru, Nanomed.-Nanotechnol., 2010, 6, 245.

2016 S. K. Smart, A. I. Cassady, G. Q. Lu, D. J. Martin, Carbon, 2006, 44, 1034.

17 A. M. Schrand, B. M. Stacy, S. Payne, L. Dosser, S. M. Hussain, ACS Appl. Mater. Interfaces, 2011, 3, 3971.

18 A. S. Burlaka Lukin, S. Prylutska, O. Remeniak, Y. Prylutsky, M. Shuba, S. Maksimenko, U. Ritter, P. Scharff, Exp. Oncol., 2010, 32, 48.

19 L. Picou, C. McMann, P. H. Elzer, F. M. Enright, A. S. Biris, D. Boldor, Nanotechnology, 2010, 21, 435101.

20 A. R. Biris, S. Ardelean, D. Lupu, I. Misan, C. Iancu, D. M. Bartos, I.

30 R. Ilie, E. Dervishi, Y. Xu, A. Biswas, A. S. Bris, Carbon, 2011, 49, 4403.

21 T. Fujigaya, T. Morimoto, N. Nakashima, Soft Matter, 2011, 7, 2647.

22 T. Shiraki, A. Dawn, T. N. Le, Y. S. Tsuchiya Tamaru, S. Shinkai, Chem. Commun., 2011, 47, 7065.

3523 E. Miyako, H. Nagata, K. Hirano, T. Hirotsu, Small, 2008, 4, 1711.

24 T. Fujigaya, T. Morimoto, Y. Niidome, N. Nakashima, Adv. Mater., 2008, 20, 3610.

25 V. E. Santo, A. M. Frias, M. Carida, R. Cancedda, M. E. Gomes, J. F. Mano, R. Reis, Biomacromolecules, 2009, 10, 1392.

4026 H. Hezaveh, I. I. Muhamad, Carbohyd. Polym., 2012, 89, 138.

27 K. H. Leong, L. Y. Chung, M. I. Noordin, K. Mohamad, M. Nishikawa, Y. Onuki, M. Morishita, K. Takayama, Carbohyd. Polym., 2011, 83, 1507.

28 Y. Liu, Y-y. Zhu, G. Wei, W-y. Lu, Eur. J. Pharm. Sci., 2009, 37, 306.

29 I. D. Rupenthal, C. R. Green, R. G. Alany, Int. J. Pharm., 2011, 411, 69.

30 A. L. Daniel-da-Silva, S. Fateixa, A. J. Guiomar, B. F. O. Costa, N. J. O. Silva, T. Trindade, B. J. Goodfellow, A. M. Gil, Nanotechnology, 2009, 20, 355602.

31 A. L. Daniel-da-Silva, L. Ferreira, A. M. Gil, T. Trindade, J. Colloid Interf. Sci., 2011, 355, 512.

32 A. Aldalbahi, M. in het Panhuis, Carbon, 2012, 50, 1197.

33 A. J. Granero, J. M. Razal, G. G. Wallace, M. in het Panhuis, J. Mater. Chem., 2010, 20, 7953.

34 A. B. González-Guerrero, E. Mendoza, E. Pellicer, F. Alsina, C. Fernández-Sánchez, L. M. Lechuga, Chem. Phys. Lett., 2008, 462, 256.

35 L. Chen, H. Xie, Y. Li, W. Yu, J. Nanomater., 2008, 1.

6036 H. S. Soedjak, Anal. Chem., 1994, 66, 4514.

37 T. K. L. Meyvis, B. G. Stubbe, M. J. Van Steenbergen, W. E. Hennink, S. C. De Smedt, J. Demeester, Int. J. Pharm, 2002 , 244, 163.

38 J. Prado-Fernández, J. A. Rodríguez-Vázquez, E. Tojo, J. M. Andrade, Anal. Chim. -Acta, 2003, 480, 23.

39 Y. Hou, J. Tang, H. Zhang, C. Qian, Y. Feng, J. Liu, ACS Nano, 2009, 3, 1057.

40 L. Piculell, in Food Polysaccharides and Their Applications, ed. A. M. Stephen, Marcel Dekker, New York, 1995, pp. 205-217.

7041 M. Iijima, T. Hatakeyama, M. Takahashi, H. Hatakeyama, Thermochimica Acta, 2007, 452, 53.
42 A. M. Salgueiro, A. L. Daniel-da-Silva, S. Fateixa, T. Trindade, Carbohyd. Polym., 2013, 91, 100.

43 A. L. Daniel-da-Silva, J. Moreira, R. Neto, A. C. Estrada, A. M. Gil, T. Trindade, Carbohyd. Polym., 2012, 87, 328.

44 M. R. Mangione, D. Giacomazza, G. Cavallaro, D. Bulone, V. Martorana, P. L. San Biagio, Biophys. Chem., 2007, 129, 18.

45 A. Vogel, V. Venugopalan, Chem. Rev., 2003, 103, 577. 


\section{SUPPLEMENTARY INFORMATION}

\section{Characterization of functionalized MWCNTs}

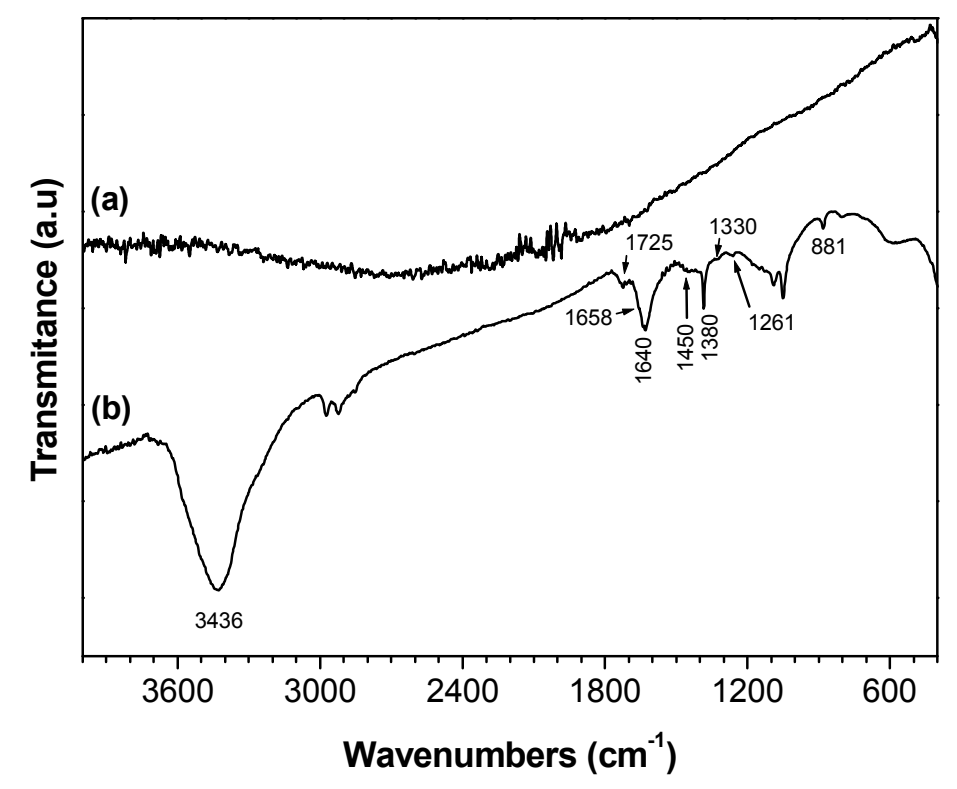

Figure S-1 FTIR-ATR spectra of (a) pristine and (b) functionalized MWCNTs.

Figure S-1 shows the FTIR spectra of the MWCNTs before and after surface functionalization. Oxygen functional groups were not detected on pristine MWCNT (Figure S-1a). In the range of $400-4000 \mathrm{~cm}^{-1}$ the functionalized MWCNT present a set of bands characteristics of oxygen functional groups (Figure S-1b): the bands at 1261, $1380,1450,1725$ and $3436 \mathrm{~cm}^{-1}$ correspond to the vibration of the groups C-O-C, C-O, $\mathrm{C}=\mathrm{C}, \mathrm{C}=\mathrm{O}$ and $\mathrm{O}-\mathrm{H}$, respectively $[\mathrm{S} 1]$ and is in agreement with the introduction of carboxylic acid groups at the surface of the MWCNTs. The sharp band at $1640 \mathrm{~cm}^{-1}$ is assigned to carbonyl of quinone type units. 
MWCNTS were further analyzed by XPS to acquire information regarding the chemical oxidation states of the resulting oxidized MWCNT comparatively with the pristine MWCNT samples. The XPS spectrum of pristine MWCNT shows only a dominant peak structure for the C 1s core level at a binding energy of 284.4 eV (peak C6 in Figure S2b), which corresponds to the graphitic carbon of the untreated MWCNT [S2]. After oxidation of MWCNTs (Figure S-2a) the C 1s peak shape changes and the broadening to high binding energies is observed, which is attributed to the presence of oxygen functionalities on the tubes. In addition, the XPS spectrum of O1s confirms the presence of oxygen functional groups on the functionalized MWCNT. The optimum curve fitting of the O1s peak for functionalized MWCNT is shown in Figure S-3 and indicates three different oxygen functionalities. The measured binding energies along with peak assignments of XPS spectra are summarized in Table S-1 and are in agreement with the values reported in literature [S2].

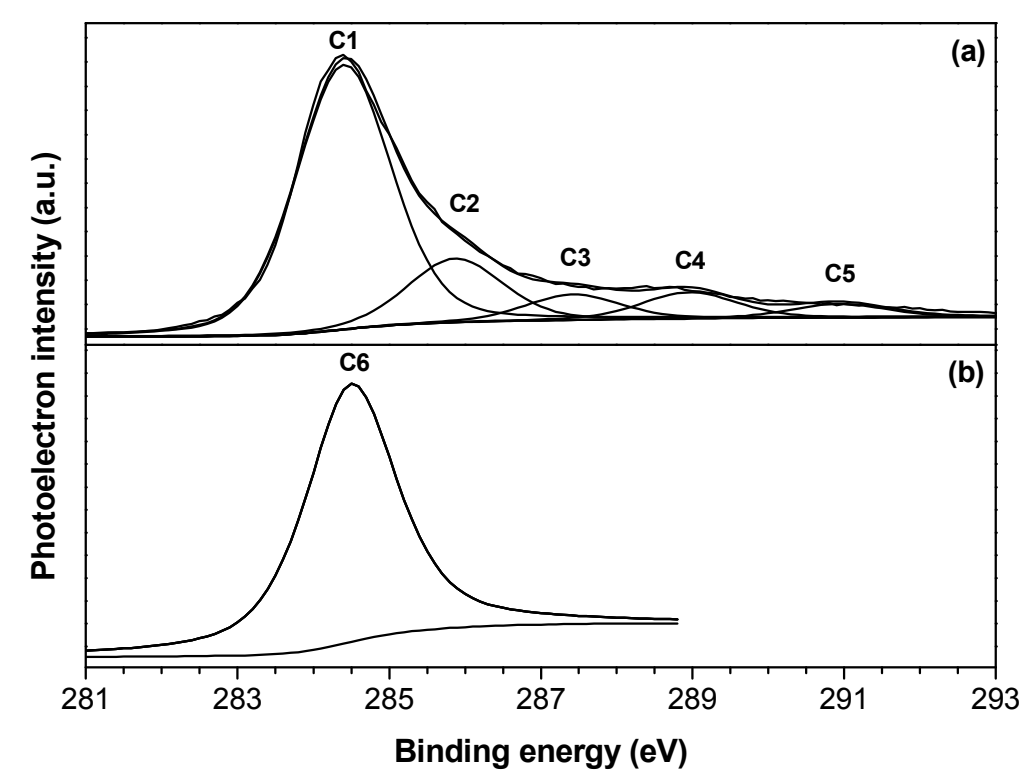

Figure S-2 XPS spectra of C 1s core levels of (a) oxidized and (b) pristine MWCNT. 


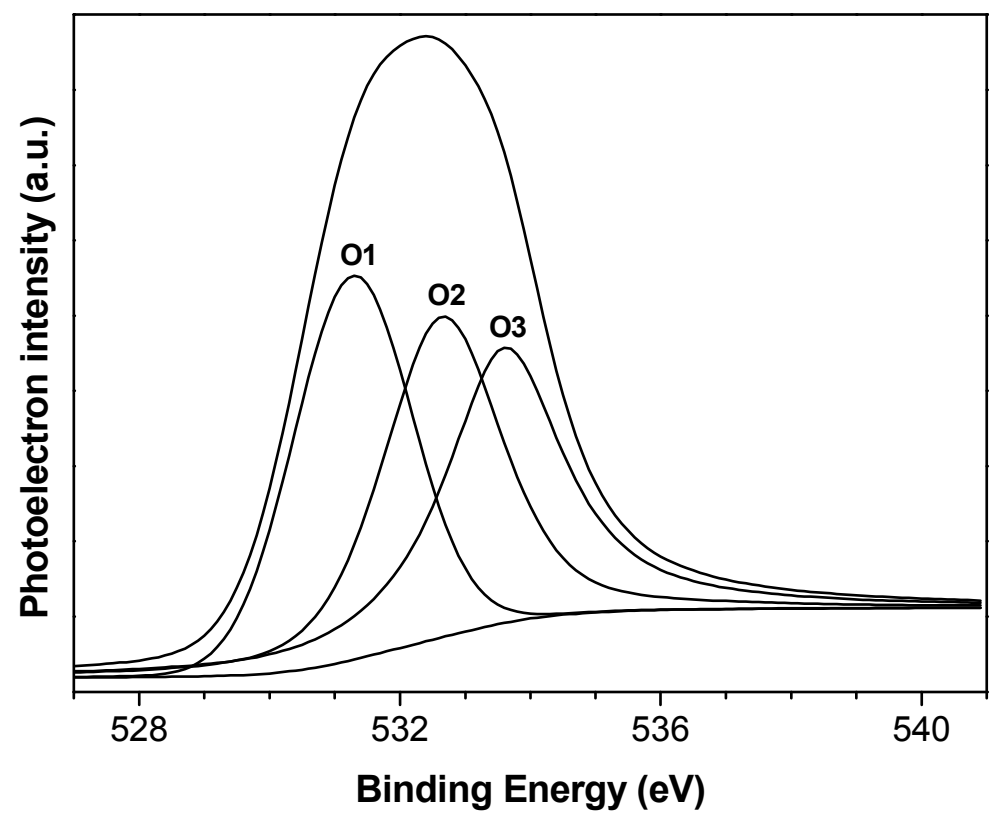

Figure S-3 XPS spectra of O 1s core levels of oxidized MWCNT.

Table S-1 XPS peak assignments of the chemical oxidation states of MWCNT of the C 1s and $\mathrm{O} 1 \mathrm{~s}$ core levels ${ }^{*}$ (Figures S3 and S4).

\begin{tabular}{lcccl}
\hline Sample & Peak & Binding energy (eV) & FWHM (eV) & Assignment \\
\hline & $\mathrm{C} 1$ & 284.4 & 1.47 & $\mathrm{C}-\mathrm{C}\left(\mathrm{sp}^{2}\right)$ \\
& $\mathrm{C} 2$ & 285.9 & 1.47 & $-\mathrm{OH}$ \\
f-MWCNT & $\mathrm{C} 3$ & 287.4 & 1.47 & $\mathrm{C}=\mathrm{O}$ \\
& $\mathrm{C} 4$ & 288.9 & 1.47 & $-\mathrm{COOH}$ \\
& $\mathrm{C} 5$ & 290 & 1.47 & $\pi \leftrightarrow \pi^{*}$ \\
& $\mathrm{O} 1$ & 531.3 & 2.13 & $\mathrm{C}=\mathrm{O}\left(\mathrm{O}_{\mathrm{G} 1}\right)$ \\
& $\mathrm{O} 2$ & 532.6 & 2.13 & $-\mathrm{OH}\left(\mathrm{O}_{\mathrm{G} 2}\right)$ \\
& $\mathrm{O} 3$ & 533.6 & 2.13 & $-\mathrm{COOH}\left(\mathrm{O}_{\mathrm{G} 4}\right)$ \\
\hline MWCNT & $\mathrm{C} 6$ & 284.5 & 1.31 & $\mathrm{C}-\mathrm{C}\left(\mathrm{sp}^{2}\right)$
\end{tabular}

"Peak centers and full widths at half-maxima (FWHM) based on curve-fitting. 


\section{MB release upon NIR light irradiation - effect of the temperature of the release medium}

The temperature of the shaker was kept at $37^{\circ} \mathrm{C}$ during all the release experiments. For the experiments performed upon exposure to NIR light the temperature of the release medium was monitored. Figure S-4 shows the evolution of the temperature of the medium during the experiments performed with the composite MWCNT $0.025 \mathrm{wt} \%$. It was found that the temperature increased up to $42^{\circ} \mathrm{C}$ in the first $3 \mathrm{~h}$ and was constant until the end of the trial (Figure S-4 - black circles). In order to evaluate the impact of this temperature increment on the MB release, an experiment of MB release was carried out following the temperature profile above described, without exposure to NIR light. The resulting release pattern (Figure S-4 - squares) was very similar to that obtained at $37^{\circ} \mathrm{C}$ without exposure to NIR light (Figure S-4 - triangles) in the first 6 hours. Conversely, the MB release carried out under irradiation of NIR light was much faster (Figure S-4 - red circles). Thus, the acceleration of the MB release from composites when exposed to NIR light cannot simply be explained by the increase of the temperature of the release medium due to the irradiation of NIR light. 


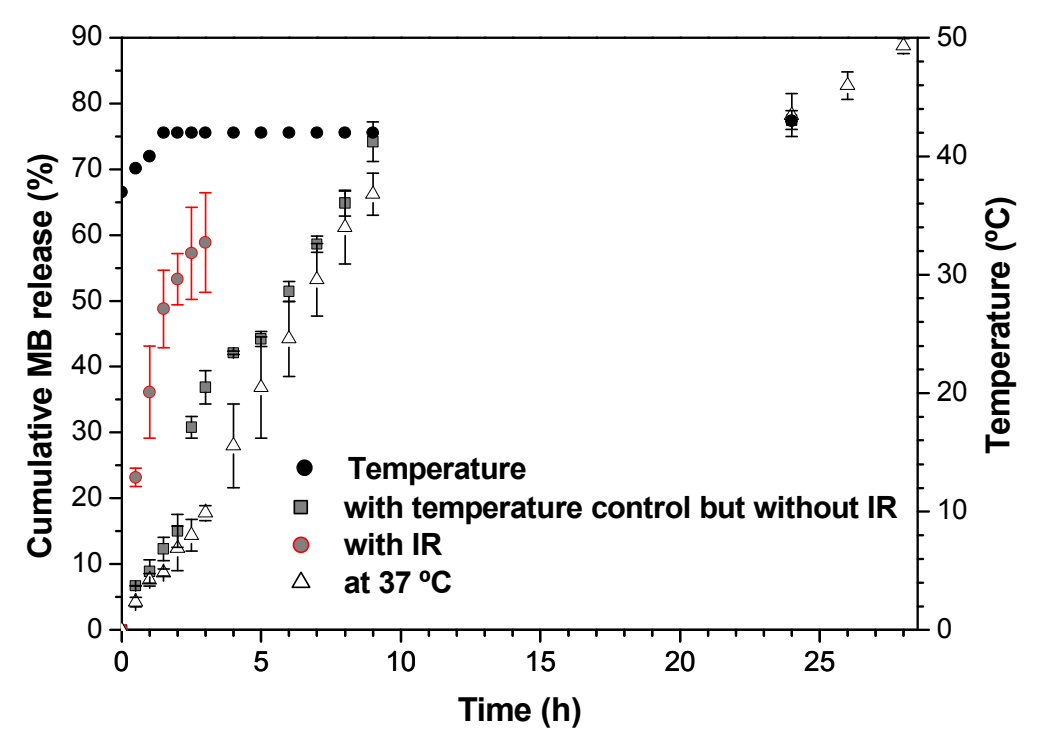

Figure S-4 In vitro MB release profiles from the composite MWCNT $0.025 \mathrm{wt} \%$ performed in different conditions.

\section{REFERENCES}

S1. (a) Grujicic M, Cao G, Rao AM, Tritt TM, Nayak S. UV-light enhanced oxidation of carbon nanotubes. Appl Surf Sci 2003; 214:289-303. (b) Coates J. In: Meyers RA. Editor Encyclopedia of analytical chemistry, Chichester; John Wiley \& Sons Ltd; 2000 p. $10815-10837$.

S2. Chiang Y-C, Lin W-H, Chang Y-C. The influence of treatment duration on multi walled carbon nanotubes functionalized by $\mathrm{H}_{2} \mathrm{SO}_{4} / \mathrm{HNO}_{3}$ oxidation. Appl Surf Sci 2011; 257:2401-10. 


\section{TABLE OF CONTENTS}
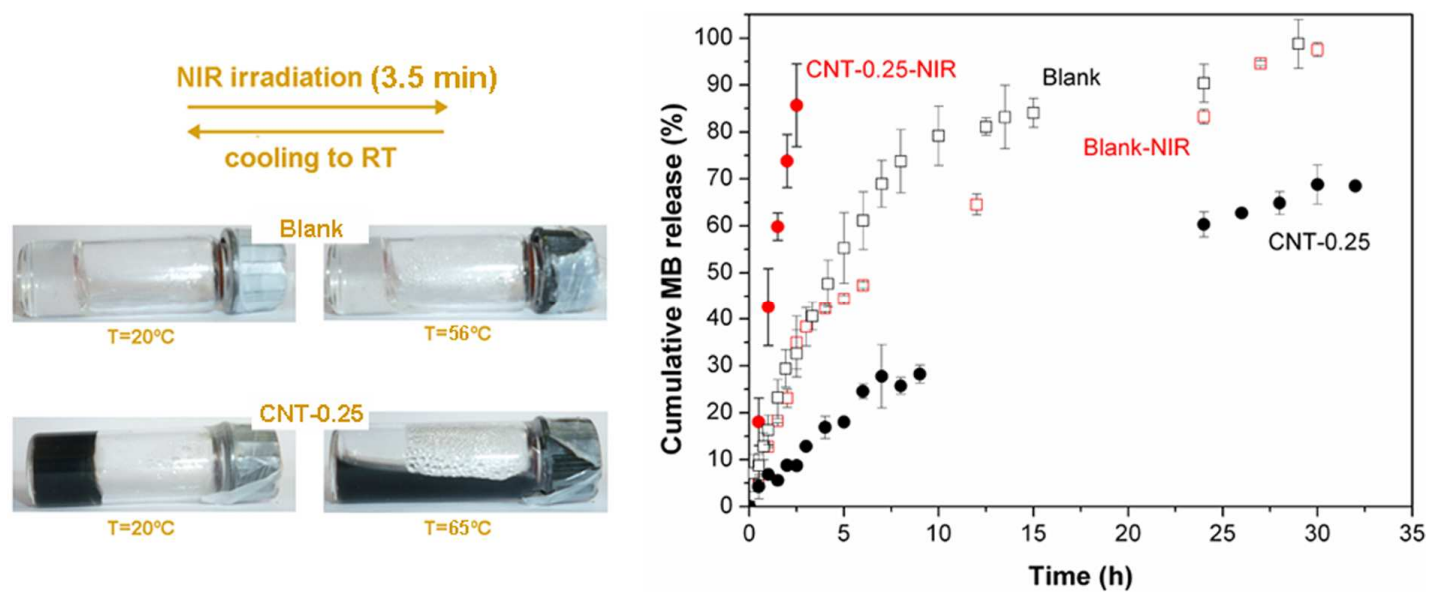

The release of a drug model from MWCNT/א-carrageenan hydrogels was induced remotely via near-infrared photothermal conversion of the MWCNTs. 